PROCEEDINGS OF THE

AMERICAN MATHEMATICAL SOCIETY

Volume 125, Number 5, May 1997, Pages 1273-1282

S 0002-9939(97)03713-1

\title{
GRÖBNER DUALITY AND MULTIPLE POINTS IN LINEARLY GENERAL POSITION
}

\author{
TEO MORA
}

(Communicated by Wolmer V. Vasconcelos)

\begin{abstract}
It is proved for each $d, 1 \leq d \leq n-1$, that a primary 0-dimensional scheme in $\mathbb{P}^{n}$ of degree $n+2+d$ in linearly general position lies in a rational normal scroll of dimension $d$.
\end{abstract}

\section{INTRODUCTION}

Gröbner, in a series of papers and in his book [4], developed a theory in order to understand both multiplicity for primary ideals at the origin and Macaulay's notion of inverse systems. Gröbner's starting point was just to generalize the obvious univariate case, where $\alpha$ is a root of $f(X)$ of multiplicity $d$ iff $\frac{\partial^{n}}{\partial X^{n}}(f)(\alpha)=0 \forall n, 0 \leq$ $n<d$; he proved that if the origin is a root of a 0 -dimensional ideal $\mathcal{J} \subset \mathcal{P}=$ $K\left[X_{1}, \ldots, X_{n}\right]$ ( $K$ any field of characteristic 0 ), then there are finitely many linear combinations $D_{i}$ of partial derivatives $\frac{\partial^{i_{1}+\cdots+i_{n}}}{\partial X_{1}^{i_{1} \ldots \partial X_{n}^{i_{n}}}}$, such that every polynomial $f \in \mathcal{J}$ satisfies $D_{i}(f)(0)=0, \forall i$. Moreover $\left\{f \in \mathcal{P}: D_{i}(f)(0)=0, \forall i\right\}$ is exactly the primary component at 0 of $\mathcal{J}$. The $D_{i}$ 's are a basis of a so called $\sigma$-stable subspace of differential conditions at 0 .

This theory by Gröbner has been systematized and its applications to polynomial system solving have been investigated in [5], [6], where it was christened Gröbner Duality. This paper proposes a different application of Gröbner Duality toward points in linearly general position.

Configurations of projective points in linearly general position (i.e. no $k+2$ of them are contained in a plane of dimension $k$ ) have been much investigated since such configurations arise by cutting a variety of dimension $d$ with a linear variety of dimension $n-d$. The old Castelnuovo result that such a configuration of $n+3$ points in $\mathbb{P}^{n}$ lies in a rational normal curve has been generalized in the context of schemes [3]; around the Castelnuovo Theory, there arose the question whether a suitable configuration lies in a rational normal scroll. In [2] it was proved for each $d, 1 \leq d \leq n-2$, that a configuration of $n+2+d$ points in $\mathbb{P}^{n}$ lies in a rational normal scroll of dimension $d$.

Let $\mathfrak{q}$ be a primary ideal at the origin in linearly general position and dimension $\mu \geq n+3$, so - by the result in [3] - of embedding dimension 1 ; let us denote by $\mathfrak{q}_{0}$, $\mathfrak{q}_{1}$ the unique primary ideals s.t. $\mathfrak{q}_{i} \supset \mathfrak{q}, \operatorname{mult}\left(\mathfrak{q}_{0}\right)=n+1, \operatorname{mult}\left(\mathfrak{q}_{1}\right)=n+3$. The

Received by the editors September 1, 1995 and, in revised form, November 5, 1995.

1991 Mathematics Subject Classification. Primary 13P10.

(C)1997 American Mathematical Society 
approach which allowed me to generalize the result of [2] to the case of primary ideals at the origin, is quite straightforward:

- Gröbner Duality allowed me to fix a suitable frame of coordinates $Y_{1}, \ldots, Y_{n}$ s.t. $\mathfrak{q}_{0}$ contains the ideal of the rational normal curve with parametric equations $Y_{i}=t^{i}, 1 \leq i \leq n$ (Prop. 11).

- This allowed me to describe the differential conditions defining $\mathfrak{q}_{0}$ (Lemma 9)

- and (by continuation) those defining $\mathfrak{q}_{1}$ and $\mathfrak{q}$ (Prop. 12);

- the basic duality of Gröbner theory then allows me to compute the ideal J defining the unique rational normal curve s.t. $\mathcal{J} \subset \mathfrak{q}_{1}$ (Prop. 16), whose existence and uniqueness are proved in [3].

- A further application of Gröbner Duality allowed me to verify that $\mathfrak{q}$ lies in a rational normal scroll of the expected dimension.

\section{Preliminaries}

2.1. Gröbner Duality. Let $\mathcal{P}=K\left[Y_{1}, \ldots, Y_{n}\right]$ and let $\mathbf{T}$ be the semigroup generated by $\left\{Y_{1}, \ldots, Y_{n}\right\}$.

$D\left(i_{1}, \ldots, i_{n}\right): \mathcal{P} \rightarrow \mathcal{P}$ defines the differential operator

$$
D\left(i_{1}, \ldots, i_{n}\right)=\frac{1}{i_{1} ! \ldots i_{n} !} \frac{\partial^{i_{1}+\cdots+i_{n}}}{\partial Y_{1}^{i_{1}} \cdots \partial Y_{n}^{i_{n}}} .
$$

This notation will however be simplified by writing $D(t):=D\left(i_{1}, \ldots, i_{n}\right)$ where $t=Y_{1}^{i_{1}} \ldots Y_{n}^{i_{n}}$; we moreover denote

$$
\mathbf{D}:=\{D(t): t \in \mathbf{T}\}, \quad \mathbf{D}^{(i)}:=\{D(t) \in \mathbf{D}: \operatorname{deg}(t) \leq i\}
$$

and $\operatorname{Span}_{K}(\mathbf{D})$ denotes the $K$-vector space generated by $\mathbf{D}$.

If $X_{1}, \ldots, X_{j} \in \mathcal{P}$ are linearly independent linear forms, the definition of $D(t)$ is obviously generalized to define $D(\tau) \in \operatorname{Span}_{K}(\mathbf{D})$, for each $\tau \in\left\langle X_{1}, \ldots, X_{j}\right\rangle$; on the basis of that we generalize the notation above to denote

$$
\begin{gathered}
\mathbf{D}\left(X_{1}, \ldots, X_{j}\right):=\left\{D(t): t \in\left\langle X_{1}, \ldots, X_{j}\right\rangle\right\}, \\
\mathbf{D}^{(i)}\left(X_{1}, \ldots, X_{j}\right):=\left\{D(t) \in \mathbf{D}\left(X_{1}, \ldots, X_{j}\right): \operatorname{deg}(t) \leq i\right\} .
\end{gathered}
$$

For each $j=1 \ldots n, \sigma_{j}=\sigma_{Y_{j}}: \operatorname{Span}_{K}(\mathbf{D}) \rightarrow \operatorname{Span}_{K}(\mathbf{D})$ is the antiderivative with respect to $Y_{j}$, i.e. the linear map s.t.:

$$
\sigma_{Y_{j}}\left(D\left(i_{1}, \ldots, i_{n}\right)\right)= \begin{cases}D\left(i_{1}, \ldots, i_{j}-1, \ldots, i_{n}\right) & \text { if } i_{j}>0 \\ 0 & \text { otherwise. }\end{cases}
$$

Since $\sigma_{Y_{j}} \sigma_{Y_{i}}=\sigma_{Y_{i}} \sigma_{Y_{j}} \forall i, j$, the linear maps $\sigma_{t}$ are obviously defined $\forall t \in \mathbf{T}$. A $K$-vector subspace $V \subset \operatorname{Span}_{K}(\mathbf{D})$ is $\sigma$-stable if $\forall t \in \mathbf{T}, \forall L \in V, \sigma_{t}(L) \in V$. If $V$ is a $\sigma$-stable subspace of $\operatorname{Span}_{K}(\mathbf{D})$, let

$$
\Im(V):=\{f \in \mathcal{P}: L(f)(0)=0 \forall L \in V\} .
$$

Let $\mathfrak{m}=\left(Y_{1}, \ldots, Y_{n}\right) \subset \mathcal{P}$; if $\mathcal{J} \subset \mathfrak{m}$ is an ideal, define

$$
\Delta(\mathcal{J}):=\left\{L \in \operatorname{Span}_{K}(\mathbf{D}): L(f)(0)=0 \forall f \in \mathcal{J}\right\} .
$$

Theorem 1. There is a one-to-one correspondence between $\mathfrak{m}$-primary ideals of $\mathcal{P}$ and finite dimensional $\sigma$-stable subspaces of $\operatorname{Span}_{K}(\mathbf{D})$. More exactly, every $\mathfrak{m}$ primary ideal $\mathfrak{q}$ corresponds to the finite dimensional $\sigma$-stable subspace $\Delta(\mathfrak{q})$, and 
every finite dimensional $\sigma$-stable subspace $V \subset \operatorname{Span}_{K}(\mathbf{D})$ corresponds to the $\mathfrak{m}$ primary ideal $\Im(V)$, so that $\mathfrak{q}=\Im(\Delta(\mathfrak{q}))$ and $V=\Delta(\Im(V))$.

Moreover $\operatorname{dim}_{K}(\Delta(\mathfrak{q}))=\operatorname{mult}(\mathfrak{q})$, $\operatorname{mult}(\Im(V))=\operatorname{dim}_{K}(V)$.

Proof. Cf. [4], pp. 174-178.

Gröbner Duality is generalizable as:

Theorem 2. $\Im$ and $\Delta$ give a one-to-one correspondence between ideals of $\mathcal{P}$ closed w.r.t. the $\mathfrak{m}$-adic topology and $\sigma$-stable subspaces of $\operatorname{Span}_{K}(\mathbf{D})$.

Let us consider now the linear map $\rho_{Y_{j}}: \operatorname{Span}_{K}(\mathbf{D}) \rightarrow \operatorname{Span}_{K}(\mathbf{D}), j=1, \ldots, n$, s.t. $\rho_{Y_{j}}\left(D\left(i_{1}, \ldots, i_{n}\right)\right)=D\left(i_{1}, \ldots, i_{j}+1, \ldots, i_{n}\right)$. Again $\forall t \in \mathbf{T}, \rho_{t}$ is defined in the obvious way and $\sigma_{t} \rho_{t}=$ Id holds $\forall t \in \mathbf{T}$.

Each $L \in \operatorname{Span}_{K}(\mathbf{D} \backslash\{I d\})$ can be uniquely written as $L=L_{1}+\cdots+L_{n}$ where

$$
L_{j} \in \operatorname{Span}_{K}\left(\mathbf{D}\left(Y_{j}, \ldots, Y_{n}\right) \backslash \mathbf{D}\left(Y_{j+1}, \ldots, Y_{n}\right)\right) .
$$

Denote $L^{\geq j}:=\sum_{i=j}^{n} L_{i}$; analogously, we use also the notation $L^{\leq j}, L^{>j}, L^{<j}$.

A semigroup ordering $<$ on $\mathbf{T}$ obviously induces an ordering also on $\mathbf{D}$; with respect to it, we define $T(L) \in \mathbf{D}$ for any $L \in \operatorname{Span}_{K}(\mathbf{D})$ as follows: if $L=\sum c_{i} D_{i}$ with $c_{i} \neq 0, D_{i} \in \mathbf{D}, D_{1}>D_{2}>\cdots$, then $T(L)=D_{1}$.

Let $U \subset \operatorname{Span}_{K}(\mathbf{D})$ be $\sigma$-stable. We then denote $T(U)=\{T(L): L \in U\}$, which is $\sigma$-stable itself, and

$$
\mathcal{C}(U):=\left\{D(\tau) \in \mathbf{D}: D(\tau) \notin T(U), \sigma_{i}(D(\tau)) \in T(U) \forall i\right\} .
$$

Definition 3. Let $D(t) \in \mathcal{C}(U)$; an element $L \in \operatorname{Span}_{K}(\mathbf{D})$ s.t.

(c1) $T(L)=D(t)$

(c2) $\forall j, \sigma_{j}(L) \in U$,

(c3) if $L=D(t)+\sum c_{i} D\left(\tau_{i}\right)$ with $c_{i} \neq 0$, then $\forall i D\left(\tau_{i}\right) \notin T(U)$

will be called a continuation of $U$ at $t$.

Proposition 4. (1) If a continuation of $U$ at $t$ exists, then there is a unique one, $C_{U, t}$, satisfying

(c4) if $C_{U, t}=D(t)+\sum c_{i} D\left(\tau_{i}\right)$ with $c_{i} \neq 0$, then for each $D\left(\tau_{i}\right) \in \mathcal{C}(U)$, there is no continuation of $U$ at $\tau_{i}$.

(2) The following conditions are equivalent:

a) $V:=U+\operatorname{Span}_{K}(\{L\})$ is $\sigma$-stable.

b) There are $t_{0}>\cdots>t_{s}, D\left(t_{i}\right) \in \mathfrak{C}(U)$ such that $L=C_{U, t_{0}}+\sum_{i=1}^{s} c_{i} C_{U, t_{i}}$.

Proof. Cf. [5], Cor. 4.1 and Prop. 4.1.

Proposition 5. Let $\Phi: \mathbf{T} \mapsto \mathbb{Z}^{r}$ be a semigroup morphism; let $\mathcal{J}_{\Phi}$ the ideal

$$
\left\{m_{1}-m_{2}: m_{i} \in \mathbf{T}, \Phi\left(m_{1}\right)=\Phi\left(m_{2}\right)\right\} \subset \mathcal{P}
$$

and $\forall \alpha \in \mathbb{Z}^{r}$, let $\delta_{\alpha}=\sum_{\substack{t \in \mathbf{T} \\ \Phi(t)=\alpha}} D(t) \in \operatorname{Span}_{K}(\mathbf{D})$. Then $\Delta\left(\mathcal{J}_{\Phi}\right)=\left\{\delta_{\alpha}: \alpha \in \mathbb{Z}^{r}\right\}$.

Proof. Let us fix any $\alpha \in \mathbb{Z}^{r}$ and let $\mathcal{P}_{\alpha}=\operatorname{Span}_{K}(\{m \in \mathbf{T}: \Phi(m)=\alpha\}), \mathcal{J}_{\alpha}=$ $\mathcal{J}_{\Phi} \cap \mathcal{P}_{\alpha}$. Obviously $\forall f \in \mathcal{J}_{\alpha}$ one has $\delta_{\alpha}(f)=0$. Also $\forall t \in \mathbf{T}$ s.t. $\Phi(t)=\alpha$ and $\forall m \in \mathbf{T}$ s.t. $\Phi(m) \neq \alpha$ we have $D(t)(m)(0)=0$, so that $\delta_{\alpha}(f)=0, \forall f \in \mathcal{J}_{\Phi}$. Since moreover $\operatorname{dim}_{K}\left(\mathcal{J}_{\alpha}\right)=\operatorname{dim}_{K}\left(\mathcal{P}_{\alpha}\right)-1$, the claim follows by a vector space dimensional count. 


\subsection{0-dim. primary ideals in l.g.p. and rational normal scrolls.}

Definition 6. Let $\mathfrak{q}$ be an $\mathfrak{m}$-primary ideal. $\mathfrak{q}$ is said to be in linearly general position (l.g.p.) if for each linear primary ideal $\wp \subset \mathfrak{m}$ we have $\operatorname{mult}(\mathfrak{q}+\wp) \leq$ $1+\operatorname{dim}(\wp)$.

Definition 7. A rational normal scroll is the variety defined by the ideal $\mathcal{R}(M) \subset \mathcal{P}$ generated by the minors of a matrix

$$
M=\left(\begin{array}{ccccc}
\ell_{11} & \ldots & \ell_{1 j} & \ldots & \ell_{1 k} \\
\ell_{21} & \ldots & \ell_{2 j} & \ldots & \ell_{2 k}
\end{array}\right)
$$

such that

- $\ell_{i j} \in \operatorname{Span}_{K}\left(\left\{1, Y_{1}, \ldots, Y_{n}\right\}\right), \forall 1 \leq i \leq 2,1 \leq j \leq k$;

- $\lambda \ell_{11}+\mu \ell_{21}, \ldots \lambda \ell_{1 j}+\mu \ell_{2 j}, \ldots \lambda \ell_{1 k}+\mu \ell_{2 k}$ are linearly independent $\forall(\lambda, \mu) \in \mathbb{P}^{2}$.

In the case of a rational normal curve $(k=n)$, I need the following fact.

Lemma 8. Let $M$ be a matrix as above $(k=n)$ and such that, moreover,

(1) $\mathcal{R}(M) \subset \mathfrak{m}$,

(2) $\mathcal{R}(M) \not \subset \mathfrak{m}^{2}$,

(3) $D\left(Y_{1}\right) \in \Delta(\mathcal{R}(M))$.

Then there are $c_{k} \in K, 1 \leq k \leq n$, and linear forms $\mathbf{l}_{i}, 2 \leq i \leq n$, s.t., denoting

$$
\mathcal{M}=\left(\begin{array}{cccccc}
Y_{1} & Y_{2} & \ldots & Y_{j} & \ldots & Y_{n} \\
1+\sum_{k=1}^{n} c_{k} Y_{k} & \mathbf{l}_{2} & \ldots & \mathbf{l}_{j} & \ldots & \mathbf{l}_{n}
\end{array}\right),
$$

one has $\mathcal{R}(M)=\mathcal{R}(\mathcal{N})$.

Proof. By 1 and 2, up to permutations and operations of rows and columns we can assume that each form $\ell_{i j}$ except $\ell_{21}$ is s.t. $\ell_{i j}(0)=0$, and $\ell_{21}(0)=1$, so that

$$
\ell_{21}=1+\sum_{k=1}^{n} \ell_{21}^{(k)} Y_{k}, \quad \ell_{i j}=\sum_{k=1}^{n} \ell_{i j}^{(k)} Y_{k} \quad \forall(i, j) \neq(2,1) .
$$

Let $M^{(\lambda \rho)}=\ell_{1 \lambda} \ell_{2 \rho}-\ell_{2 \lambda} \ell_{1 \rho}$ be the minor over the $\lambda^{\text {th }}$ and $\rho^{\text {th }}$ columns $(\lambda<\rho)$. By 3 we have

$$
0=D\left(Y_{1}\right)\left(M^{(1 \rho)}\right)(0)=\ell_{11}^{(1)} \ell_{2 \rho}(0)+\ell_{11}(0) \ell_{2 \rho}^{(1)}-\ell_{21}^{(1)} \ell_{1 \rho}(0)-\ell_{21}(0) \ell_{1 \rho}^{(1)}=-\ell_{1 \rho}^{(1)},
$$

which implies $\ell_{1 \rho}^{(1)}=0$ for $\rho>1$. Since $\left\{\ell_{1 \rho}, \rho=2, \ldots, n\right\}$ are linearly independent, column operations can be used to modify the first column so that $\ell_{11}=Y_{1}$. For the same reason, further column operations not affecting the first column can now allow us to assume that $\ell_{1 j}=Y_{j}$.

\section{Relations}

Let us impose a second graduation $w t$ on $\mathcal{P}$ by setting $w t\left(Y_{i}\right)=i$, and let us denote $\mathcal{R}=\left(Y_{2}-Y_{1}^{2}, \ldots, Y_{n}-Y_{1}^{n}\right)$ and $\forall j: \mathbf{D}(j)=\left\{t \in \mathbf{D}\left(Y_{1}, \ldots, Y_{j-1}\right)\right.$ : $w t(t)=j\}, \delta_{j}=\sum_{\substack{t \in \mathbf{T} \\ w t(t)=j}} D(t), \delta_{j}^{\prime}=\sum_{t \in \mathbf{D}(j)} D(t), U_{j}=\operatorname{Span}_{K}\left(\delta_{0}, \ldots, \delta_{j}\right)$. We also fix as semigroup ordering $<$ on $\mathbf{T}$ the lexicographical ordering s.t. $Y_{1}>Y_{2}>\ldots>Y_{n}$.

Lemma 9. The following hold:

(1) $\Delta(\mathcal{R})=\left\{\delta_{j}: 0 \leq j\right\}$. 
(2) $\sigma_{i}\left(\delta_{j}\right)=\delta_{j-i}, \forall i, j: i \leq j$.

(3) $\forall j, T\left(\delta_{j}\right)=Y_{1}^{j}$.

(4) $\forall j, \mathcal{C}\left(U_{j}\right)=\left\{D\left(Y_{1}^{j+1}\right), D\left(Y_{2}\right), \ldots, D\left(Y_{n}\right)\right\}$.

(5) The generic continuations of $U_{j-1}$ are $\delta_{j}^{\prime}+\sum_{k=2}^{n} c_{k} D\left(Y_{k}\right)$.

(6) $\forall j, \delta_{j}=\sum_{i=1}^{j} \rho_{i}\left(\delta_{j-i}^{\geq i}\right)$.

Proof. 1 is a corollary of Proposition 5; 2, 3 and 6 require just an obvious verification; 3 implies $T\left(U_{j}\right)=\left\{1, Y_{1}, \ldots, Y_{1}^{j}\right\}$ which is equivalent to 4 .

Ad 5: First of all remark that $\delta_{j}^{\prime}$ is a continuation: c1) and c3) are obvious and c2) follows from 2. Then 5 follows from this, Prop. 4 and the obvious fact that $D\left(Y_{k}\right), k>1$, is a continuation.

Corollary 10. Let $\mathfrak{q}$ be an $\mathfrak{m}$-primary ideal of multiplicity $\mu=j+1, j \leq n$, and embedding dimension 1 and such that $\Delta(\mathfrak{q}) \cap \operatorname{Span}_{K}\left(\mathbf{D}^{(1)}\right)=\left\{I d, D\left(Y_{1}\right)\right\}$. If $U_{j-1} \subset \Delta(\mathfrak{q})$, then $\Delta(\mathfrak{q})=U_{j-1}+\operatorname{Span}_{K}(\{\delta\})$, where $\delta=\delta_{j}^{\prime}+\sum_{k=2}^{n} c_{k} D\left(Y_{k}\right)$ for some $c_{k}$.

Proof. Since $\operatorname{dim}_{K}(\Delta(\mathfrak{q}))=j+1=\operatorname{dim}_{K}\left(U_{j-1}\right)+1$ and $U_{j-1} \subset \Delta(\mathfrak{q})$, it follows that $\Delta(\mathfrak{q})=U_{j-1}+\operatorname{Span}_{K}(\{\delta\})$; then $\delta$ is a continuation of $U_{j-1}$. If $T(\delta)=D\left(Y_{l}\right)$ we have $\Delta(\mathfrak{q}) \cap \operatorname{Span}_{K}\left(\mathbf{D}^{(1)}\right)=\left\{I d, D\left(Y_{1}\right), \delta\right\}$, which implies that the embedding dimension of $\mathfrak{q}$ is 2 (cf. [1], prop. 2) and gives a contradiction. So $T(\delta)=D\left(Y_{1}^{\mu}\right)$. The claim then follows from this, Prop. 4 and Lemma 9.5.

Proposition 11. Let $\mathfrak{q} \subset K\left[X_{1}, \ldots, X_{n}\right]$ be an $\mathfrak{m}$-primary ideal of multiplicity $\mu$ and s.t. its embedding dimension is 1 .

The following conditions are then equivalent:

(1) $\mathfrak{q}$ is in l.g.p.;

(2) there is a coordinate frame $Y_{1}, \ldots, Y_{n}$ s.t.

$$
\mathfrak{q}= \begin{cases}\left(Y_{1}^{\mu}, Y_{2}-Y_{1}^{2}-Y_{1}^{n+1} f_{2}\left(Y_{1}\right), \ldots, Y_{n}-Y_{1}^{n}-Y_{1}^{n+1} f_{n}\left(Y_{1}\right)\right) & \text { if } \mu>n, \\ \left(Y_{1}^{\mu}, Y_{2}-Y_{1}^{2}, \ldots, Y_{\mu-1}-Y_{1}^{\mu-1}, Y_{\mu}, \ldots, Y_{n}\right) & \text { if } \mu \leq n ;\end{cases}
$$

(3) there is a coordinate frame $Y_{1}, \ldots, Y_{n}$ s.t., setting $\nu=\min (n, \mu-1)$ :

- $\forall i, 1 \leq i \leq \nu, V_{i}:=\Delta(\mathfrak{q}) \cap \operatorname{Span}_{K}\left(\mathbf{D}^{(i)}\left(Y_{1}, \ldots, Y_{i}\right)\right)=U_{i}$

- $D\left(Y_{i}\right)\left(Y_{j}\right)=0$ iff $1 \leq i \leq \nu<j \leq n$.

Proof. $1 \Longrightarrow 3$. Since $\mathfrak{q}$ has embedding dimension 1, then ([1], prop. 2) there is $Y_{1}$ s.t. $V_{1}$ is generated by $\left\{I d, D\left(Y_{1}\right)\right\}$. Now let us assume that for some $i<\nu$ we have found linearly independent forms $Y_{1}, \ldots, Y_{i}$ s.t. $V_{i}=U_{i}$ (this holds at least for $i=1$, giving the basis for the induction) and let us fix linear forms $Z_{i+1}, \ldots, Z_{n}$ s.t. $\mathfrak{m}=\left(Y_{1}, \ldots, Y_{i}, Z_{i+1}, \ldots, Z_{n}\right)$. Our aim is to prove that there is a further linear form $Y_{i+1}$ linearly independent with $Y_{1}, \ldots, Y_{i}$ s.t. $V_{i+1}=U_{i+1}$, which is sufficient to prove the statement by the inductive argument, since the vector space of the linear forms $Z$ s.t. $D\left(Y_{i}\right)(Z)=0 \forall i \leq \nu$ is orthogonal to $\operatorname{Span}_{K}\left(Y_{1}, \ldots, Y_{\nu}\right)$.

To prove that, notice that, $\operatorname{as}_{K} \operatorname{dim}_{K}(\Delta(\mathfrak{q}))=\operatorname{mult}(\mathfrak{q})=\mu>i+1=\operatorname{dim}\left(U_{i}\right)$, there is $\delta \in \Delta(\mathfrak{q}) \backslash U_{i}$ s.t. $\delta$ is a continuation of $U_{i}$; then by Corollary 10

$$
\delta=\delta_{i+1}^{\prime}+\sum_{k=2}^{i} c_{k} D\left(Y_{k}\right)+\sum_{k=i+1}^{n} c_{k} D\left(Z_{k}\right) \text { for some } c_{k} .
$$


Let us denote $Y_{i+1}:=\sum_{k=2}^{i} c_{k} D\left(Y_{k}\right)+\sum_{k=i+1}^{n} c_{k} D\left(Z_{k}\right)$. To prove that $Y_{i+1} \notin$ $\operatorname{Span}_{K}\left(\left\{Y_{1}, \ldots, Y_{i}\right\}\right)$, let us consider $\wp=\left(Z_{i+1}, \ldots, Z_{n}\right)$; then $\Delta(\mathfrak{q}+\wp)=\Delta(\mathfrak{q}) \cap$ $\Delta(\wp)=\Delta(\mathfrak{q}) \cap \operatorname{Span}_{K}\left(\mathbf{D}\left(Y_{1}, \ldots, Y_{i}\right)\right)=V_{i}$.

Therefore if $Y_{i+1} \in \operatorname{Span}_{K}\left(\left\{Y_{1}, \ldots, Y_{i}\right\}\right)$, then $\left\{\delta_{0}, \ldots, \delta_{i}, \delta\right\} \subset \Delta(\mathfrak{q}+\wp)$, so that $\operatorname{mult}(\mathfrak{q}+\wp)=\operatorname{dim}_{K}(\Delta(\mathfrak{q}+\wp))>i+1$, against the assumption that $\mathfrak{q}$ is in l.g.p.

We then have $\delta=D\left(Y_{i+1}\right)+\delta_{i+1}^{\prime}=\delta_{i+1}$, so that $V_{i+1} \supseteq U_{i+1}$. Moreover if $V_{i+1} \neq U_{i+1}$ then it would contain a continuation L of $U_{i+1}$; according to Lemma $9, T(L)=D\left(Y_{1}^{i+2}\right)$, so $L \notin \mathbf{D}^{(i+1)}$. By this contradiction, we conclude that $V_{i+1}=U_{i+1}$.

$3 \Longleftrightarrow 2$. Since $\operatorname{Span}_{K}\left(\mathbf{D}^{(i)}\right)=\Delta\left(\mathfrak{m}^{i+1}\right)$, Lemma 9.1 implies that for $i \leq n$

$$
U_{i}=\operatorname{Span}_{K}\left(\delta_{0}, \ldots, \delta_{i}\right)=\operatorname{Span}_{K}\left(\left\{\delta_{j}, j \geq 0\right\}\right) \cap \operatorname{Span}_{K}\left(\mathbf{D}^{(i)}\right)=\Delta\left(\mathcal{R}+\mathfrak{m}^{i+1}\right),
$$

so that

$$
\begin{aligned}
& \operatorname{Span}_{K}\left(\delta_{0}, \ldots, \delta_{i}\right) \subset \Delta(\mathfrak{q}) \\
& \Longleftrightarrow \mathfrak{q} \subset \mathcal{R}+\mathfrak{m}^{i+1}= \begin{cases}\left(Y_{1}^{n+1}, Y_{2}-Y_{1}^{2}, \ldots, Y_{n}-Y_{1}^{n}\right) & \text { if } i=n, \\
\left(Y_{1}^{i+1}, Y_{2}-Y_{1}^{2}, \ldots, Y_{i}-Y_{1}^{i}, Y_{i+1}, \ldots, Y_{n}\right) & \text { if } i<n ;\end{cases}
\end{aligned}
$$

which is of course equivalent to 2 , since $\mathfrak{q}$ has embedding dimension 1.

$2 \Longrightarrow 1$. Let $\mathfrak{q}=\left(Y_{1}^{\mu}, Y_{2}-Y_{1}^{2}-Y_{1}^{n+1} f_{2}\left(Y_{1}\right), \ldots, Y_{n}-Y_{1}^{n}-Y_{1}^{n+1} f_{n}\left(Y_{1}\right)\right)$ and let $\wp$ be a linear ideal thru the origin. Let $k<n$ be the maximal index s.t. $\wp \subset\left(Y_{k+1}, \ldots, Y_{n}\right)$. Then

$$
\begin{gathered}
\mathfrak{q}+\wp \subset\left(Y_{1}^{\mu}, Y_{2}-Y_{1}^{2}-Y_{1}^{n+1} f_{2}\left(Y_{1}\right), \ldots, Y_{n}-Y_{1}^{n}-Y_{1}^{n+1} f_{n}\left(Y_{1}\right), Y_{k+1}, \ldots, Y_{n}\right) \\
=\left(Y_{1}^{\mu}, Y_{1}^{k+1}, Y_{2}-Y_{1}^{2}, \ldots, Y_{k}-Y_{1}^{k}, Y_{k+1}, \ldots, Y_{n}\right),
\end{gathered}
$$

so its multiplicity is at most $k+1$ while the dimension of $\wp$ is at least $k$.

\section{Differential CONDitions OF L.G.P. m-PRIMARY IDEALS}

As a consequence of Prop. 11, if $\mathfrak{q}$ is an $\mathfrak{m}$-primary ideal of multiplicity $\mu>n+1$, of embedding dimension 1 and in l.g.p., we can assume that it is contained in $\mathfrak{q}_{0}=\Im\left(\operatorname{Span}_{K}\left(\left\{\delta_{0}, \ldots, \delta_{n}\right\}\right)\right)$ and that a basis of $\mathfrak{q}$ is $\mathcal{B}=\left(Y_{1}^{\mu}, f_{2}, \ldots, f_{n}\right)$, where

$$
f_{j}=Y_{j}-Y_{1}^{j}-\sum_{i=1}^{\mu-n-1} d_{j i} Y_{1}^{n+i} \text {. }
$$

Our aim is now to describe $\Delta(\mathfrak{q})$ in terms of $d_{j i}$.

Proposition 12. $\Delta(\mathfrak{q})=\left\{L_{0}, \ldots, L_{n}, L_{n+1}, \ldots, L_{\mu-1}\right\}$, where

$$
\begin{gathered}
L_{i}=\delta_{i} \forall i \leq n, \\
L_{n+1}=\delta_{n+1}+\sum_{j=2}^{n} d_{j 1} D\left(Y_{j}\right), \\
L_{n+2}=\delta_{n+2}+\sum_{j=2}^{n} d_{j 1} D\left(Y_{1} Y_{j}\right)+\sum_{j=2}^{n} d_{j 2} D\left(Y_{j}\right),
\end{gathered}
$$


and in general

$$
L_{n+k}=\sum_{j=1}^{n} \rho_{j}\left(L_{n+k-j}^{\geq j}\right)+\sum_{j=2}^{n} \sum_{i=1}^{k} d_{j i} \rho_{j}\left(L_{k-i}^{\geq j}\right) .
$$

Proof. One has to prove that:

(1) $L_{n+k}(f)(0)=0$ for each $f \in \mathcal{B}$;

(2) $\sigma_{j}\left(L_{n+k}\right) \in \operatorname{Span}_{K}(\Delta(\mathfrak{q}))$ for each $j$.

Ad 1: remark that $L_{n+k}=D\left(Y_{1}^{n+k}\right)+\tilde{L}_{n+k}+\sum_{j=2}^{n} d_{j k} D\left(Y_{j}\right)$, where $\tilde{L}_{n+k}$ is a combination of terms $D\left(Y_{i} t\right)$ s.t. $i>1, t \neq 1$, so that $\tilde{L}_{n+k}(f)(0)=0 \forall f \in \mathcal{B}$. It is immediate to verify that $D\left(Y_{1}^{n+k}\right)\left(f_{i}\right)(0)=-d_{i k}=-\sum_{j=2}^{n} d_{j k} D\left(Y_{j}\right)\left(f_{i}\right)(0)$.

Ad 2: A direct computation allows us to verify that:

- $\sigma_{1}\left(L_{n+k}\right)=L_{n+k-1}$

- $\sigma_{l}\left(L_{n+k}\right)=L_{n+k-l}+\sum_{i=1}^{k} d_{l i} L_{k-i}, l>1$.

\section{Normal RATIONAL CURVES CONTAINING L.G.P. m-PRIMARY IDEALS}

This section will deal with the following

Problem. Given an l.g.p. $\mathfrak{m}$-primary ideal $\mathfrak{q}_{1} \subset \mathcal{P}$ s.t. $\operatorname{mult}\left(\mathfrak{q}_{1}\right)=\mu=n+3$, explicitly construct the unique rational normal curve J s.t. $\mathfrak{q}_{1} \supset \mathcal{J}$.

The existence of $\mathcal{J}$ is assured by [3], Th. 1 . Of course such a construction does not give at all a proof of [3], Th. 1; in fact, our construction is based on the fact that we can fix a frame of coordinates satisfying conditions 2 and 3 of Prop. 11; it is [3], Th. 1 itself which guarantees the existence of such a frame since it lets us apply Prop. 11 guaranteeing that $\mathfrak{q}_{1}$ has embedding dimension 1 .

As a consequence of Lemma 8 , we know that if $\mathcal{J}$ is a rational normal curve there is a matrix $\mathcal{M}$ as in (1) s.t. $\mathcal{R}(\mathcal{N})=\mathcal{J}$. In the rest of the argument we will write $\mathbf{l}_{j}=\sum_{k=1}^{n} \mathbf{l}_{j}^{(k)} Y_{k}$ for $\mathbf{j} \geq 2$, we will set $\mathbf{l}_{1}=1+\sum_{k=1}^{n} c_{k} Y_{k}$ and we will denote by $\mathcal{M}_{\mu \nu}, \forall \mu, \nu$ the matrices

$$
\mathcal{M}_{\mu \nu}=\left(\begin{array}{cccc}
D\left(Y_{\mu}\right)\left(Y_{1}\right) & D\left(Y_{\mu}\right)\left(Y_{2}\right) & \ldots & D\left(Y_{\mu}\right)\left(Y_{n}\right) \\
D\left(Y_{\nu}\right)\left(\mathbf{l}_{1}\right) & D\left(Y_{\nu}\right)\left(\mathbf{l}_{2}\right) & \ldots & D\left(Y_{\nu}\right)\left(\mathbf{l}_{n}\right)
\end{array}\right) .
$$

For a $2 \times n$ matrix $M$ we will again write $M^{(\lambda \rho)}$ to denote the minor over the $\lambda^{\text {th }}$ and $\rho^{\text {th }}$ columns.

As a preliminary step, let us consider an l.g.p. $\mathfrak{m}$-primary ideal $\mathfrak{q}_{0}$ s.t. $\operatorname{mult}\left(\mathfrak{q}_{0}\right)=$ $n+1$ and try to compute all the rational normal curves passing thru it. We first fix a frame of coordinates $Y_{1}, \ldots, Y_{n}$ such that conditions 2 and 3 are satisfied by $\mathfrak{q}_{0}$.

Remark 13. (1) $D\left(Y_{\mu} Y_{\nu}\right)\left(\mathcal{M}^{(\lambda \rho)}\right)(0)=\mathcal{M}_{\mu \nu}^{(\lambda \rho)}+\mathcal{M}_{\nu \mu}^{(\lambda \rho)}$.

(2) $\mathcal{R}(\mathcal{M}) \subset \mathfrak{q}_{0} \Longleftrightarrow \delta_{j}\left(\mathcal{M}^{(\lambda \rho)}\right)(0)=0 \forall \lambda, \rho, j=1 \ldots, n$

$$
\Longleftrightarrow \delta_{j}^{*}\left(\mathcal{M}^{(\lambda \rho)}\right)(0)=0 \forall \lambda, \rho, j=1 \ldots, n
$$

where $\delta_{j}^{*}$ is the projection of $\delta_{j}$ over $\operatorname{Span}_{K}\left(\mathbf{D}^{(2)}\right)$, i.e.

$$
\delta_{i}^{*}=D\left(Y_{i}\right)+\sum_{j \leq i / 2} D\left(Y_{j} Y_{i-j}\right) .
$$

(3) $\delta_{1}^{*}\left(\mathcal{M}^{(\lambda \rho)}\right)(0)=0 \forall \lambda, \rho$. 


$$
\begin{aligned}
& \text { (4) } 0=\delta_{2}^{*}\left(\mathcal{M}^{(\lambda \rho)}\right)(0)= \begin{cases}\mathbf{l}_{2}^{(1)}-1 & \text { if }(\lambda, \rho)=(1,2), \\
\mathbf{l}_{j}^{(1)} & \text { if }(\lambda, \rho)=(1, j), 2<j, \\
0 & \text { otherwise }\end{cases} \\
& \text { (5) } D\left(Y_{i+1}\right)\left(\mathcal{M}^{(\lambda \rho)}\right)(0)= \begin{cases}-1 & \text { if }(\lambda, \rho)=(1, i+1), \\
0 & \text { otherwise }\end{cases}
\end{aligned}
$$

Proposition 14. The following conditions are equivalent:

(1) $\mathcal{R}(\mathcal{M}) \subset \mathfrak{q}_{0}$;

(2) $\forall j, 2 \leq j \leq n$ there is $d_{j}$ s.t. $\mathbf{l}_{j}=Y_{j-1}+\sum_{k=j}^{n-1} c_{k-j+1} Y_{k}+d_{j} Y_{n}$.

Proof. Clearly $2 \Longrightarrow 1$ is immediate, so let us focus on the proof of $1 \Longrightarrow 2$. The argument goes by induction on $i$ : one assumes that:

$$
\forall k<i \quad \mathbf{l}_{j}^{(k)}= \begin{cases}0 & \text { if } k<j-1, \\ 1 & \text { if } k=j-1, \\ c_{k-j+1} & \text { if } j \leq k<i,\end{cases}
$$

and proves the case $\mathbf{A}(i+1)$; note that $\mathbf{A}(2)$ is guaranteed by Rem. 13.4. As the case $\mathbf{A}(2)$ was derived by computing $\delta_{2}^{*}\left(\mathcal{M}^{(\lambda \rho)}\right)(0)$, we will deduce the case $\mathbf{A}(i+1)$ computing $\delta_{i+1}^{*}\left(\mathcal{M}^{(\lambda \rho)}\right)(0)$. Under the inductive assumption $\mathbf{A}(i)$ one has

$$
0=\delta_{i+1}^{*}\left(\mathcal{M}^{(\lambda \rho)}\right)(0)= \begin{cases}\mathbf{l}_{\rho}^{(i)}-c_{i+1-\rho} & \text { if } \lambda=1, \rho \leq i, \\ \mathbf{l}_{i+1}^{(i)}-1 & \text { if } \lambda=1, \rho=i+1, \\ \mathbf{l}_{\rho}^{(i)} & \text { if } \lambda=1, \rho>i+1, \\ 0 & \text { otherwise }\end{cases}
$$

whence we obtain $\mathbf{A}(i+1)$ and so the claim.

Let us now assume we have an l.g.p. $\mathfrak{m}$-primary ideal $\mathfrak{q}_{1}$ s.t. $\operatorname{mult}\left(\mathfrak{q}_{1}\right)=n+3$, and let us try to compute the rational normal curve passing thru it. Since $\mathfrak{q}_{1}$ has embedding dimension 1 , we know it is contained in a unique $\mathfrak{m}$-primary ideal $\mathfrak{q}_{0} \supset \mathfrak{q}_{1}$ s.t. $\operatorname{mult}\left(\mathfrak{q}_{0}\right)=n+1$.

The knowledge of $\mathfrak{q}_{0}$ allows us to fix a frame $Y_{1}, \ldots, Y_{n}$ and to compute a matrix $\mathcal{M}$ s.t. the rational normal curve $\mathcal{R}(\mathcal{N})$ contains $\mathfrak{q}_{0}$ (Prop. 14).

Remark 15. A quadratic polynomial $f$ is in $\mathfrak{q}$ if and only if $L^{*}(f)(0)=0 \forall L \in \Delta(\mathfrak{q})$, where $L^{*}$ denotes the projection of $L$ over $\operatorname{Span}_{K}\left(\mathbf{D}^{(2)}\right)$.

Proposition 16. In the above notation the only rational normal curve containing $\mathfrak{q}_{1}$ is $\mathcal{R}(\mathbf{M})$, where $\mathbf{M}$ is the matrix

$$
\left(\begin{array}{ccccc}
Y_{1} & \ldots & Y_{j} & \ldots & Y_{n} \\
Y_{0}+\sum_{i=2}^{n} c_{i} Y_{i} & \ldots & Y_{j-1}+\sum_{i=j+1}^{n} c_{i+1-j} Y_{i}+d_{j 1} Y_{n} & \ldots & Y_{n-1}+d_{n 1} Y_{n}
\end{array}\right)
$$

and $c_{i}, 2 \leq i \leq n$, are functions of $d_{j 1}$ and $d_{j 2}$ given by

$$
\left\{\begin{array}{l}
\sum_{j=3}^{n} d_{j 1} c_{j-1}+d_{n 1} d_{21}-c_{n}-d_{22}=0, \\
d_{\rho-1}+\sum_{j=\rho+1}^{n} d_{j 1} c_{j+1-\rho}+d_{n 1} d_{\rho 1}-c_{n+2-\rho}-d_{\rho 2}=0, \quad \rho>2 .
\end{array}\right.
$$


Proof. To prove the statement we have to compute which conditions are imposed on the minors $\mathcal{M}^{(\lambda \rho)}$ by $L_{n+1}, L_{n+2}$, i.e. we have just to solve the equations

$$
L_{i}^{*}\left(\mathcal{M}^{(\lambda \rho)}\right)(0)=0, \quad i=n+1, n+2,1 \leq \lambda<\rho \leq n,
$$

where

$$
\begin{gathered}
L_{n+1}^{*}=\sum_{j=1}^{n / 2} D\left(Y_{j} Y_{n+1-j}\right)+\sum_{i=2}^{n} d_{i 1} D\left(Y_{i}\right), \\
L_{n+2}^{*}=\sum_{j=2}^{n / 2} D\left(Y_{j} Y_{n+2-j}\right)+\sum_{i=2}^{n} d_{i 1} D\left(Y_{1} Y_{i}\right)+\sum_{i=2}^{n} d_{i 2} D\left(Y_{i}\right) .
\end{gathered}
$$

A direct computation shows that

$$
L_{n+1}^{*}\left(\mathcal{M}^{(\lambda \rho)}\right)(0)=0, \forall \lambda, \rho \Longrightarrow \mathbf{l}_{\rho}^{(n)}=c_{n-\rho+1}+d_{\rho 1}, \forall \rho \geq 2
$$

After substituting these values in $\mathcal{M}$, note that the coefficient of $Y_{i}$ in $\mathcal{M}_{2 i}$ is $c_{1}$, so we can subtract the first row from the second $c_{1}$ times and obtain the matrix $\mathbf{M}$. Moreover $L_{n+2}^{*}\left(\mathbf{M}^{(\lambda \rho)}\right)(0)=0, \forall \lambda, \rho$ gives $(* *)$.

\section{Normal RATiOnal SCROLlS CONTAINING L.G.P. m-PRIMARY IDEALS}

It is quite natural to continue the same computation we did in the previous paragraph, basing ourself on Proposition 12, to study where an l.g.p. m-primary ideal $\mathfrak{q}$ with $\operatorname{mult}(\mathfrak{q})=\mu>n+3$ lies.

We already know that $\Delta(\mathfrak{q})=\left\{L_{0}, \ldots, L_{n}, L_{n+1}, \ldots, L_{\mu-1}\right\}$ and that for $\mathbf{M}$ defined as in Prop. 16 we have $\mathcal{R}(\mathbf{M}) \subset \Im\left(\operatorname{Span}_{K}\left(\left\{L_{0}, \ldots, L_{n+2}\right\}\right)\right)=\mathfrak{q}_{1}$, so we have just to compute $L_{j}\left(\mathbf{M}^{(\lambda \rho)}\right)$ to derive our conclusions. To follow this plan we need to introduce some notation and fix some details.

Remark 17. For each $\lambda>1, \mathbf{M}^{(\lambda \rho)}$ does not depend on $Y_{0}, \ldots, Y_{\lambda-2}$ nor on the terms $Y_{\lambda-1}^{2}, Y_{\lambda-1} Y_{\lambda}$ nor on linear terms $Y_{j}$. In order not to write operators which are clearly zero, let us therefore denote by $\mathbf{D}^{[\lambda]}$ the set of elements

$$
\mathbf{D}^{[\lambda]}=\left\{D\left(Y_{i} Y_{j}\right): i \leq j, i \geq \lambda\right\} \cup\left\{D\left(Y_{\lambda-1} Y_{j}: j>\lambda\right\}\right.
$$

and for $L \in \operatorname{Span}_{K}(\mathbf{D})$ let us denote by $L^{[\lambda]}$ its projection over $\operatorname{Span}_{K}\left(\mathbf{D}^{[\lambda]}\right)$; then $\forall L \in \operatorname{Span}_{K}(\mathbf{D}), L\left(\mathbf{M}^{(\lambda \rho)}\right)=L^{[\lambda]}\left(\mathbf{M}^{(\lambda \rho)}\right)$.

With the same notation of Prop. 16, a straightforward computation allows to verify that:

Lemma 18. For $3 \leq k \leq n$

$$
\begin{aligned}
L_{n+k}^{[k-1]}= & \sum_{i=k}^{\frac{n+k}{2}} D\left(Y_{i} Y_{n+k-i}\right)+\sum_{j=k}^{n} d_{j 2} D\left(Y_{k-2} Y_{j}\right) \\
& +\sum_{j=k-1}^{n} d_{j 1} D\left(Y_{k-1} Y_{j}\right)+d_{k-1}{ }_{1} D\left(Y_{k-1}^{2}\right) .
\end{aligned}
$$

Proposition 19. Let $\mathbf{M}$ be the matrix of Proposition 16. Then $L_{n+k}^{[k-1]}\left(\mathbf{M}^{(\lambda \rho)}\right)=0$ for $3 \leq k \leq n, \lambda \geq k-1$. 
Proof. At this point it is just a question of explicitly computing $L_{n+k}^{[k-1]}\left(\mathbf{M}^{(\lambda \rho)}\right)$, obtaining

$$
\begin{cases}d_{k-1} 1+\sum_{j=k+1}^{n} d_{j 1} c_{j+1-k}+d_{k 1} d_{n 1}-c_{n+2-k}-d_{k 2}=0 & \text { if } \lambda=k-1, \rho=k, \\ d_{\rho-1}+\sum_{j=\rho+1}^{n} d_{j 1} c_{j+1-\rho}+d_{\rho 1} d_{n 1}-c_{n+2-\rho}-d_{\rho 2}=0 & \text { if } \lambda=k-1, \rho>k, \\ c_{n+k-\lambda-\rho+1}-c_{n+k-\lambda-\rho+1}=0 & \text { if } \lambda>k-1,\end{cases}
$$

where the expressions for $L_{n+k}^{[k-1]}\left(\mathbf{M}^{(k-1 \rho)}\right)$ are 0 because of the equalities (**) of Proposition 16.

Corollary 20. If $\mathfrak{q}$ is a primary ideal at the origin in l.g.p. of multiplicity $\mu=$ $n+2+d, 1 \leq d \leq n-1$, then it lies in a rational normal scroll of dimension $d$.

Proof. In fact, in an appropriate frame of coordinates, the dual basis of $\mathfrak{q}$ is

$$
\left\{\delta_{0}, \ldots, \delta_{n}, L_{n+1}, \ldots, L_{n+d+1}\right\} .
$$

Let $\mathcal{S}$ be the ideal generated by the minors of the matrix containing only the last $n-d+1$ columns of $\mathbf{M}$; by Prop. 19, $\mathcal{S} \subset \mathfrak{q}$.

\section{ACKNOWLEDGMENT}

I want to thank M.E. Rossi for pointing out this problem and helping me through the algebraic geometry behind it, M.G. Marinari for suggestions, and GAGE, École Polytechnique, Palaiseau in Paris for hospitality and financial support during the preparation of this paper.

\section{REFERENCES}

[1] E. Becker, M.G. Marinari, T. Mora, C. Traverso, The shape of the shape lemma, Proc. ISSAC'94, ACM, New York, 1994, pp. 129-133.

[2] M.P. Cavaliere, M.E. Rossi, G. Valla, Quadrics through a set of points and their syzygies, Math. Z. 218 (1995), 25-42. MR 96a:13016

[3] D. Eisenbud, J. Harris, Finite projective schemes in linearly general position, J. Algebraic Geometry 1 (1992), 15-30. MR 92i:14035

[4] W. Gröbner, Algebraische Geometrie II, Bibliogr. Inst., Mannheim, 1970. MR 48:8499

[5] M.G. Marinari, T.Mora, H.M. Möller, On multiplicities in polynomial system solving, Trans. Amer. Math. Soc. 348 (1996), 3283-3321. MR 96k:13039.

[6] - Gröbner duality and multiplicities in polynomial system solving, Proc. ISSAC'95 (C. Traverso, ed.), ACM, New York, 1995, pp. 167-180.

Dima and Disi, Università di Genova, Viale Dodecaneso 35, 16146 Genova, Italy

E-mail address: theomora@dima.unige.it 